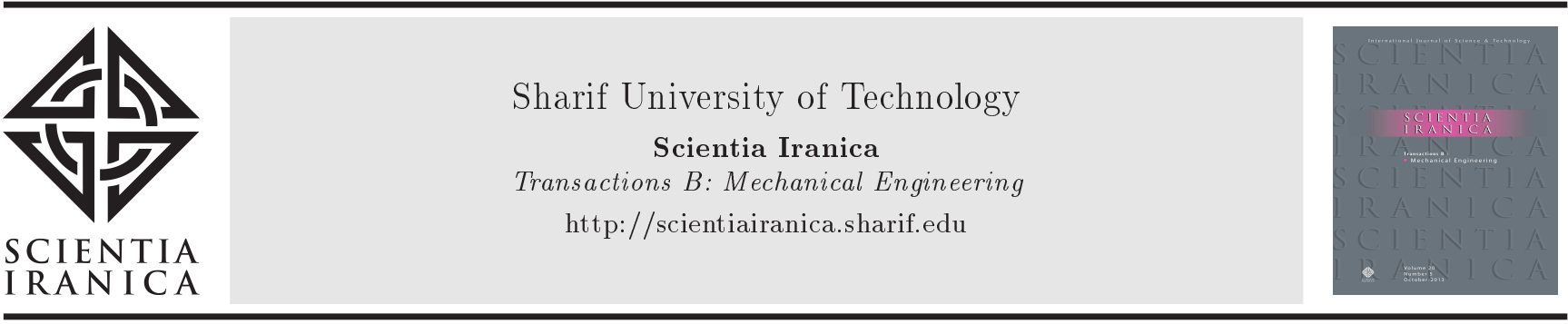

\title{
Nonlinear vibration analysis of a partially coated circular microplate under electrostatic actuation
}

\author{
R. Sepahvandi, M. Zamanian*, B. Firouzi, and S.A.A. Hosseini \\ Department of Mechanical Engineering, Faculty of Engineering, Kharazmi University, Tehran, P.O. Box 15719-14911, Iran.
}

Received 26 November 2017; received in revised form 21 October 2018; accepted 8 December 2018

\section{KEYWORDS}

Electrostatic

actuation;

Circular microplate;

Static deflection;

Primary resonance;

Perturbation theory.

\begin{abstract}
This paper investigates the optimal configuration of a partially two-layered circular capacitive microplate subjected to AC-DC electrostatic actuation. To this end, the static deflection due to DC electrostatic actuation, natural frequency of vibration about static position, and primary resonance response due to $\mathrm{AC}$ electrostatic actuation are studied. Primarily, the nonlinear equations of motion are derived through Classical Laminated Plate Theory (CLPT). Then, the static position and natural frequency of vibration around the static position are obtained using Galerkin approach. The forced vibration equations around the static position are separated using Galerkin method and solved by the multiple scale perturbation theory. Firstly, the impact of changes in the second-layer radius on the variations of static and dynamic responses of the system is studied while its thickness remains constant. Then, the effect of changes in the second-layer thickness is studied while its radius remains constant. Finally, the impact of simultaneous changes in the radius and thickness of the second layer is studied while its volume remains constant. The results show that the highest frequency and lowest static deflection occur when the second layer covers fifty percent of the first layer. This result can be used for designing high-speed microsensors.

(C) 2020 Sharif University of Technology. All rights reserved.
\end{abstract}

\section{Introduction}

Microelectronic integrated circuits can be considered as the thinking minds of systems, and microelectromechanical systems develop this thinking power with the addition of eye and arm to them so that microsystems can sense and control the surrounding environment. The main element of many microelectromechanical systems, e.g., micropumps, microphones, and microsensors, is a circular microplate under electrostatic actuation [1]. Electrostatic actuation is generated by applying electrical voltage between a microplate and a fixed electrode plate, being on the opposite side. In

*. Corresponding author. Tel.: +982634579600

E-mail address: zamanian@khu.ac.ir (M. Zamanian)

doi: $10.24200 /$ sci. 2018.5649 .1399 resonance microsensor applications, a combination of $\mathrm{AC}$ and DC voltage is used. In these configurations, the microplate deflects to the static position due to DC electrostatic actuation and, then, AC actuation make the system oscillate around that position. In this system, DC voltage serves as a regulator of system sensitivity and natural frequency. The voltage in which a microplate becomes unstable and connects to an opposite fixed electrode plate is called pull-in voltage [2]. Many studies have been conducted for modeling and calculating the static deflection, pullin voltage, natural frequency, and dynamic response of a system. The most important of such studies are addressed below.

Vogl and Nayfeh [3] established equations governing the clamped circular plate and, then, discretized the system by using Galerkin approach. They solved the equations in the equilibrium states due to a 
general electric potential and determined the natural frequencies of the axisymmetric modes for the stable deflected position. In another work, they investigated the response of the electrically actuated clamped circular plate to the primary resonance excitation of its first axisymmetric mode using an analytical reducedorder model [4]. Liao et al. [5] demonstrated that the ratio of the dynamic to static pull-in voltages for a clamped circular microplate was approximately $92 \%$. Further to that, when the squeezed-film effect induced by the air gap between the two plates was taken into account, the value of the aforementioned ratio increased slightly. An electrostatically actuated microplate was modeled by Bertarelli et al. [6] for micropump applications. Wang et al. [7] analyzed the thermal and size effects on the nonlinear vibration of the electrostatically actuated circular microplate. It was shown in this work that the geometrically nonlinear strain had significant effect on the frequency for the large initial gap to thickness ratio of the plate. The free vibration of symmetric circular Fiber Metal Laminated (FML) hybrid plates was studied by Shooshtari and Dalir [8]. They obtained the governing equation of motion by considering von Kármán nonlinearity and using First-order Shear Deformation Theory (FSDT). Khorshidi et al. [9] analyzed static pull-in instability and natural frequency of circular and annular plates in the electrical field. They studied the effect of rigid core, radial load, geometric nonlinearity, and inner radius on the pull-in instability of circular plates. Karimzadeh et al. [10] studied the size-dependent dynamic behavior of circular rings on elastic foundation using modified couple stress theory. The mechanical behavior of a capacitive microphone with a clamped circular diaphragm was studied by Dowlati et al. [11] using modified couple stress theory. They obtained the governing equation of motion by using Kirchhoff thin plate theory. Afterwards, they used an SSLM method to linearize the equation. At last, they applied Galerkin method to solve the equations.

The nonlinear vibration of a rotating annular disc made of Functionally Graded Material (FGM) with variable thicknesses was investigated by Shahriari et al. [12]. They examined the natural frequencies and critical speed of the rotating FG annular disk with two types of boundary conditions.

Jallouli et al. [13] proved how the von Kármán nonlinearity and the plate imperfections could lead to a significant delay in pull-in occurrence. Saghir et al. [14] studied the static and dynamic behaviors of an imperfect plate under electrostatic actuation. They used von Kármán to obtain the governing equation. A reducedorder model based on Galerkin method was used to simulate the mechanical behavior of the microplate. They used experimental data to validate their work.

In some other studies, the pull-in instability and the vibration for the elliptic and circular electrostatically actuated microplates were investigated, considering the Casimir force [15-18]. Zhang [19] used the principle of virtual work to conduct the large deflection of a circular plate. He proposed a new approximate analytical solution to study the plate-membrane transition behavior of the deflection. Medina et al. [20,21] studied the behavior of initially curved circular microplates under electrostatic actuation. Their model was based on Kirchhoff's hypothesis and the nonlinear von Kármán straindisplacement relations. Caruntu and Oyervides [22,23] studied the resonance of an electrostatically actuated circular plate consisting of deformable and conductive circular plates in the presence of surface force. They showed that pull-in occurred at a relatively large AC voltage. They used the perturbation method to study the nonlinear parametric resonance. In another study, the effects of mechanical shock on the stability and dynamic response of an Micro-Electro-Mechanical System (MEMS) circular capacitive microphone were investigated [24]. It was shown that mechanical shock loads could impose considerable noise on the microphone's response. The mechanical behavior of a circular FGM micro-plate subjected to electrostatic force and mechanical shock was studied by Sharafkhani et al. [25]. They used step-by-step linearization and reduced-order approach based on Galerkin method to solve the nonlinear equation of the static deflection and dynamic motion. Shabani et al. [26] investigated the dynamic behavior of a circular microplate interacting with compressible fluid and excited by electrostatic force. They utilized Kirchhoff's thin plate theory for the actuating microplate, considered the operating fluid as inviscid, and derived the eigenvalue problem of the coupled system using Fourier-Bessel expansion. In some work, the mechanical behavior of an electrostatically actuated circular microplate in the presence of hydrostatic force was investigated. $\mathrm{Li}$ et al. [27] studied the effects of both electrostatic force and uniform hydrostatic pressure on the resonant frequency of a clamped circular microplate. Nabian et al. [28] evaluated the instability of a circular plate under non-uniform electrostatic pressure and uniform hydrostatic pressure. They linearized the governing equations and solved them through the finite difference method. Tian-Jie [29] studied the behavior the electrostatically actuated circular microplate in the presence of uniform hydrostatic pressure before and after the pull-in. He used a two-fold method of bisection based on the shooting method to solve differential equations. Moreover, He investigated the effect of different parameters on pull-in and validated their results based on the results obtained in the previous studies. The dynamic pull-in instability and free vibration of circular microplates subjected to combined 
hydrostatic and electrostatic forces were investigated by taking into account size effect based on the strain gradient elasticity theory [30,31]. Rashvand et al. [32] considered the effect of the intrinsic length scale on the stability and fundamental frequency of a fully clamped circular microplate, which can be used as an RF MEMS resonator. A modified couple stress theory was utilized to model the microplate with respect to the variable length-scale parameter. In [33], a closed-form solution was presented for in-plane and out-of-plane free vibrations of simply supported Functionally Graded (FG) rectangular micro/nanoplates. Further, the dynamic response of a circular clamped microplate actuated by a DC/AC non-linear electrostatic coupling force was considered while taking into account the residual stress, hydrostatic pressure acting on the upper surface, and squeeze-film damping generated by the air gap between the vibrating microplate and fixed substrate [34].

Despite the studies mentioned above, the mechanical behavior of a partially two-layered circular microplate under electrostatic actuation has not been examined so far, which is addressed in this study. This configuration is important in two respects. The natural frequency in an MEMS device is an indication of the system performance speed. Therefore, it is investigated how the natural frequency of an electrostatically actuated microplate can be increased by compositing it partially. Moreover, pull-in and nonlinear shift of resonance frequency are undesirable phenomena for the MEMS devices. Therefore, this study examines how these phenomena can be optimized by compositing the microplate as partial. To this end, the nonlinear equations of motion are derived through Classical Laminated Plate Theory (CLPT). In the previous study [35], it was shown that the application of the CLPT method was an acceptable method that resulted in an almost exact outcome that matched experimental data. Consequently, CLPT is used in this work to analyze the behavior of microplates.

Then, the static position and natural frequency of vibration around static position are obtained using
Galerkin approach. The linear mode shapes of the non-uniform microplate, i.e., a microplate coated as partial by the second layer, are used as comparison functions. The forced vibration equations around the static position are separated using Galerkin method and solved by the multiple scale perturbation theory. The impacts of the changes in the second-layer radius and thickness while assuming that the radius and thickness remain constant on the mechanical behavior of the system are investigated. At the end, effects of simultaneous changes in the radius and thickness of the second layer, with the assumption that its volume remains constant, are considered on the static and dynamic responses of the system.

\section{Modeling and formulation}

The system consists of a circular microplate with the radius $R_{o}$ under clamped boundary conditions. Another layer with the radius $R_{i}$ coats a part of the first layer. The plate is made of silicon. Electrostatic voltage of $V_{d}+V_{a} \cos (\hat{\Omega} \hat{t})$ is applied between the microplate and the fixed electrode plate, which is placed at the distance $d$ from it. In this relation, $\hat{t}$ is time, $V_{d}$ is the DC voltage, and $V_{a}$ and $\hat{\Omega}$ are the amplitude and frequency of AC voltage, respectively. Figure 1 shows an overall view of the studied system.

The system consists of two sections considering Figure 1. In Section 1, a microplate is two layered, and it is one layered in Section 2. By applying Newton's second law to the sheared element shown in (Figure 2), the equation of motion in the first section is as follows [25]:

$$
\begin{aligned}
& \frac{d N_{\hat{r}}}{d \hat{r}}+\frac{N_{\hat{r}}+N_{\theta}}{\hat{r}}=0, \\
& \frac{d Q_{z}}{d \hat{r}}+\frac{Q_{z}}{\hat{r}}+p+\left(\rho_{1} h_{1}+\rho_{2} h_{2}\right) \frac{d^{2} \hat{w}_{1}}{d \hat{t}^{2}}=0, \\
& Q_{z}=\frac{d M_{\hat{r}}}{d \hat{r}}+\frac{M_{\hat{r}}-M_{\theta}}{\hat{r}},
\end{aligned}
$$

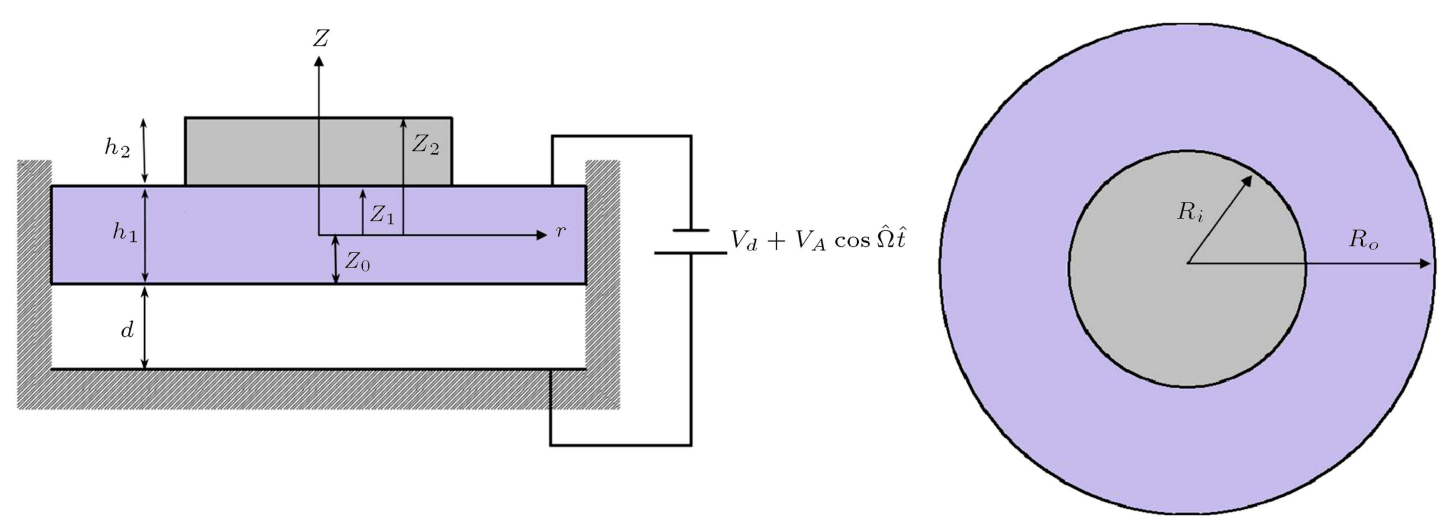

Figure 1. Overall view of a two-layered microplate under AC-DC electrostatic actuation. 

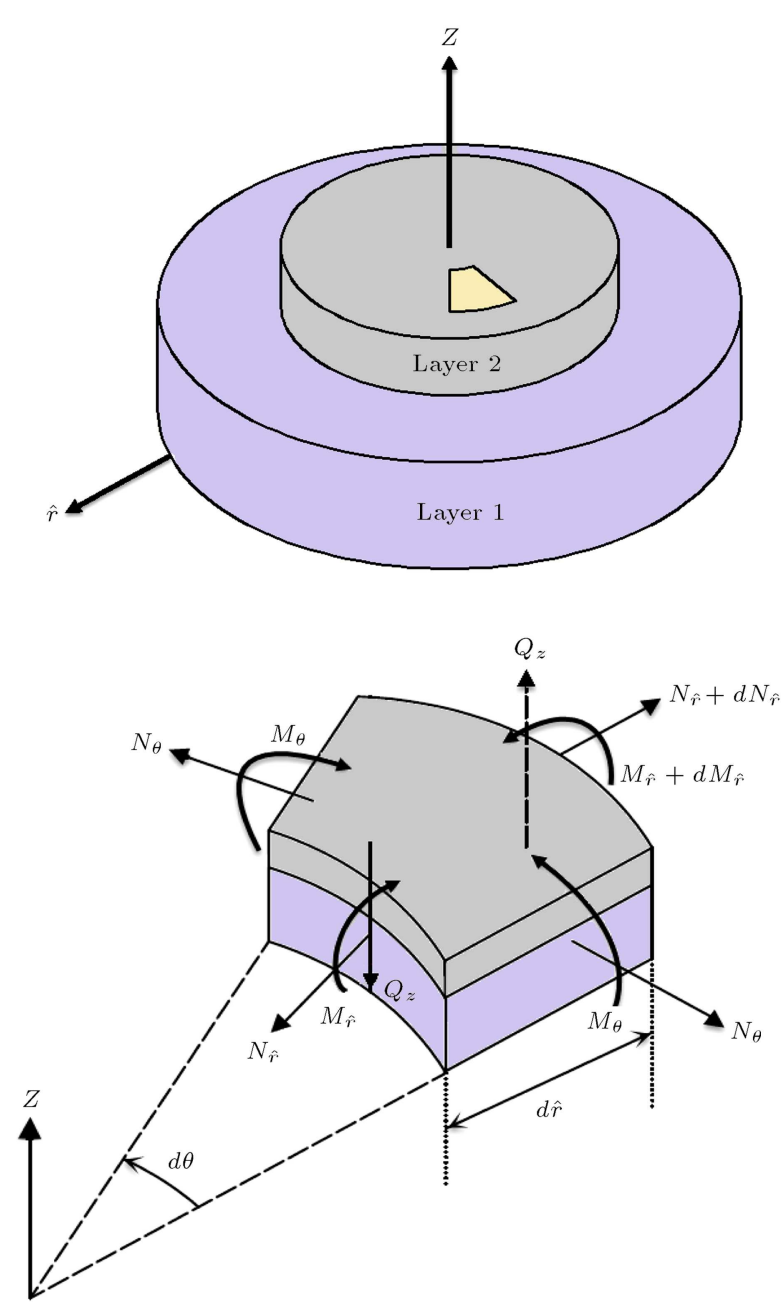

Figure 2. Free body diagram for an element of the microplate in a two-layered section.

where $\hat{w}$ indicates the transverse deflection of the microplate in the two-layered section, $\hat{r}$ is the radius position of element, $Q_{z}$ is the shear force, $N_{\hat{r}}$ and $N_{\theta}$ are the components of net force in the radial and tangential directions, $M_{\hat{r}}$ and $M_{\theta}$ are the components of net moment in the radial and tangential directions, $\rho_{1}$ is the density of the first layer, $\rho_{2}$ is the mass density of the second layer, $h_{1}$ is the thickness of the first layer, $h_{2}$ is the thickness of the second layer, and $p$ indicates the electrostatic force defined as follows [1]:

$$
p=\frac{\left(V_{d}+V_{a} \cos (\hat{\Omega} \hat{t})\right)^{2}}{2\left(d+\hat{w}_{1}\right)^{2}}
$$

Kirchhoff theory is used to calculate moment, normal force, and shearing force of the cross-section of the system. By considering the middle area of the first layer as the referenced layer and using CLPT theory for a balanced isotropic plate, the strain in the crosssection of a microplate along the radius may be defined through the following formula:

$$
\left\{\begin{array}{c}
\varepsilon_{\hat{r} \hat{r}} \\
\varepsilon_{\theta \theta}
\end{array}\right\}=\left\{\begin{array}{l}
\varepsilon_{\hat{r} \hat{r}}^{0} \\
\varepsilon_{\theta \theta}^{0}
\end{array}\right\}+z\left(\left\{\begin{array}{l}
\kappa_{\hat{r}}^{0} \\
\kappa_{\theta}^{0}
\end{array}\right\}\right)
$$

where $z$ is the distance from the reference layer. Moreover, $\varepsilon_{\hat{r} \hat{r}}$ and $\varepsilon_{\theta \theta}$ are the radial and tangential strains in the point with distance $z$ from the referenced layer; besides, $\varepsilon_{\hat{r} \hat{r}}^{0}\left(\varepsilon_{\theta \theta}^{0}\right)$ and $\kappa_{\hat{r}}^{0}\left(\kappa_{\theta}^{0}\right)$ are the strain and curvature in the radial (tangential) direction, respectively, which are calculated as follows:

$$
\begin{aligned}
& \left\{\begin{array}{c}
\varepsilon_{\hat{r} \hat{r}}^{0} \\
\varepsilon_{\theta \theta}^{0}
\end{array}\right\}=\left\{\begin{array}{c}
\frac{\partial u_{0}(\hat{r})}{\partial \hat{r}} \\
\frac{u_{0}(\hat{r})}{\hat{r}}
\end{array}\right\}, \\
& \left\{\begin{array}{c}
\kappa_{\hat{r}}^{0} \\
\kappa_{\theta}^{0}
\end{array}\right\}=\left\{\begin{array}{l}
-\frac{\partial^{2} \hat{w}_{1}(\hat{r})}{\partial \hat{r}^{2}} \\
-\frac{1}{\hat{r}} \frac{\partial \hat{w}_{1}(\hat{r})}{\partial \hat{r}}
\end{array}\right\},
\end{aligned}
$$

where $u_{0}$ indicates the deflection along the radius. Showing the stress in the radial and tangential directions for the $k$ th layer by $\sigma_{\hat{r} \hat{r}}^{k}$ and $\sigma_{\theta \theta}^{k}$, respectively, one can write the following:

$$
\begin{array}{r}
\left\{\begin{array}{c}
\sigma_{\hat{r} \hat{r}}^{k} \\
\sigma_{\theta \theta}^{k}
\end{array}\right\}=\left[Q_{k}\right]\left\{\begin{array}{c}
\varepsilon_{\hat{r} \hat{r}}^{k} \\
\varepsilon_{\theta \theta}^{k}
\end{array}\right\}=\left[Q_{k}\right]\left(\left\{\begin{array}{c}
\varepsilon_{\hat{r} \hat{r}}^{0} \\
\varepsilon_{\theta \theta}^{0}
\end{array}\right\}\right. \\
\left.+z\left\{\begin{array}{c}
\kappa_{\hat{r}}^{0} \\
\kappa_{\theta}^{0}
\end{array}\right\}\right), \quad k=1,2,
\end{array}
$$

where:

$$
Q_{k}=\frac{E_{k}}{\left(1-\nu_{k}\right)^{2}}\left[\begin{array}{cc}
1 & \nu_{k} \\
\nu_{k} & 1
\end{array}\right], \quad k=1,2,
$$

where $E_{k}$ is the elasticity module of layers 1 and 2, and $\nu_{k}$ is Poisson's ratio for them. Considering the definitions of strain and stress in Eqs. (1) to (5), the net force $N$ and moment $M$ are defined as follows:

$$
\begin{aligned}
& N=\left\{\begin{array}{l}
N_{\hat{r}} \\
N_{\theta}
\end{array}\right\}=\sum_{k=1}^{2} \int_{z_{k-1}}^{z_{k}}\left\{\begin{array}{l}
\sigma_{\hat{r} \hat{r}}^{k} \\
\sigma_{\theta \theta}^{k}
\end{array}\right\}, \\
& d z=A\left\{\begin{array}{l}
\varepsilon_{\hat{r} \hat{r}}^{0} \\
\varepsilon_{\theta \theta}^{0}
\end{array}\right\}+B\left\{\begin{array}{c}
\kappa_{\hat{r}}^{0} \\
\kappa_{\theta}^{0}
\end{array}\right\}, \\
& M=\left\{\begin{array}{l}
M_{\hat{r}} \\
M_{\theta}
\end{array}\right\}=\sum_{k=1}^{2} \int_{z_{k-1}}^{z_{k}}\left\{\begin{array}{l}
\sigma_{\hat{r} \hat{r}}^{k} \\
\sigma_{\theta \theta}^{k}
\end{array}\right\}, \\
& z d z=B\left\{\begin{array}{l}
\varepsilon_{\hat{r} \hat{r}}^{0} \\
\varepsilon_{\theta \theta}^{0}
\end{array}\right\}+F\left\{\begin{array}{l}
\kappa_{\hat{r}}^{0} \\
\kappa_{\theta}^{0}
\end{array}\right\} .
\end{aligned}
$$


Considering that $z_{0}=-\frac{h_{1}}{2}, z_{1}=\frac{h_{1}}{2}$, and $z_{2}=h_{2}+z_{1}$, the coefficient matrices of $A, B$, and $F$ are defined as follows:

$$
\begin{aligned}
A & =\left[\begin{array}{ll}
A_{11} & A_{12} \\
A_{21} & A_{22}
\end{array}\right]=\sum_{k=1}^{2} \int_{z_{k-1}}^{z_{k}}\left[Q_{k}\right] d z, \\
B & =\left[\begin{array}{ll}
B_{11} & B_{12} \\
B_{21} & B_{22}
\end{array}\right]=\sum_{k=1}^{2} \int_{z_{k-1}}^{z_{k}}\left[Q_{k}\right] z d z, \\
F & =\left[\begin{array}{ll}
F_{11} & F_{12} \\
F_{21} & F_{22}
\end{array}\right]=\sum_{k=1}^{2} \int_{z_{k-1}}^{z_{k}}\left[Q_{k}\right] z^{2} d z .
\end{aligned}
$$

By combining Eqs. (1), (3), (4), and (7), one obtains the following:

$$
\begin{aligned}
& {\left[\frac{\partial^{2} u_{0}(\hat{r})}{\partial \hat{r}^{2}}+\frac{1}{\hat{r}} \frac{\partial u_{0}(\hat{r})}{\partial \hat{r}}-\frac{u_{0}(\hat{r})}{\hat{r}}\right] A_{11}} \\
& \quad+\left[-\frac{\partial^{3} \hat{w}_{1}(\hat{r})}{\partial \hat{r}^{3}}-\frac{1}{\hat{r}} \frac{\partial^{2} \hat{w}_{1}(\hat{r})}{\partial \hat{r}^{2}}+\frac{1}{\hat{r}^{2}} \frac{\partial \hat{w}_{1}(\hat{r})}{\partial \hat{r}}\right] B_{11}=0, \\
& \quad\left[\frac{\partial^{2} u_{0}(\hat{r})}{\partial \hat{r}^{2}}+\frac{1}{\hat{r}} \frac{\partial u_{0}(\hat{r})}{\partial \hat{r}}-\frac{u_{0}(\hat{r})}{\hat{r}}\right] B_{11} \\
& \quad+\left[-\frac{\partial^{3} \hat{w}_{1}(\hat{r})}{\partial \hat{r}^{3}}-\frac{1}{\hat{r}} \frac{\partial^{2} \hat{w}_{1}(\hat{r})}{\partial \hat{r}^{2}}+\frac{1}{\hat{r}^{2}} \frac{\partial \hat{w}_{1}(\hat{r})}{\partial \hat{r}}\right] F_{11}=Q_{z} .
\end{aligned}
$$

By combining Eqs. $(9), u_{0}(\hat{r})$ is omitted and the equation of deflection governing of the system is as follows:

$$
\begin{aligned}
& {\left[-\frac{\partial^{3} \hat{w}_{1}(\hat{r})}{\partial \hat{r}^{3}}-\frac{1}{\hat{r}} \frac{\partial^{2} \hat{w}_{1}(\hat{r})}{\partial \hat{r}^{2}}+\frac{1}{\hat{r}^{2}} \frac{\partial \hat{w}_{1}(\hat{r})}{\partial \hat{r}}\right]\left(\frac{A_{11} F_{11}-B_{11}^{2}}{A_{11}}\right)} \\
& =Q_{z} .
\end{aligned}
$$

By substituting Eq. (10) into Eq. (1) and adding the effect of viscous damping, the equation of motion governing the two-layered section of the microplate will be as follows:

$$
\begin{aligned}
& {\left[\frac{\partial^{4} \hat{w}_{1}(\hat{r}, \hat{t})}{\partial \hat{r}^{4}}+\frac{2}{\hat{r}} \frac{\partial^{3} \hat{w}_{1}(\hat{r}, \hat{t})}{\partial \hat{r}^{3}}-\frac{1}{\hat{r}^{2}} \frac{\partial^{2} \hat{w}_{1}(\hat{r}, \hat{t})}{\partial \hat{r}^{2}}+\frac{1}{\hat{r}^{3}} \frac{\partial \hat{w}_{1}(\hat{r}, \hat{t})}{\partial \hat{r}}\right]} \\
& \quad\left(\frac{A_{11} F_{11}-B_{11}^{2}}{A_{11}}\right)+\hat{c} \frac{\partial \hat{w}_{2}(\hat{r}, \hat{t})}{\partial \hat{t}} \\
& +\left(\rho_{1} h_{1}+\rho_{2} h_{2}\right) \frac{d^{2} \hat{w}_{2}}{d \hat{t}^{2}} \\
& \quad=\frac{\left(V_{d}+V_{a} \cos (\hat{\Omega} \hat{t})\right)^{2}}{2\left(d+\hat{w}_{2}\right)^{2}},
\end{aligned}
$$

where $\hat{c}$ is the coefficient of viscous damping. By setting $h_{2}=0$ and replacing $\hat{w}_{1}$ by $\hat{w}_{2}$ in Eq. (1), the equation of motion in the one-layered section of the microplate will be as follows:

$$
\begin{aligned}
\frac{E_{1} h_{1}^{3}}{2} & {\left[\frac{\partial^{4} \hat{w}_{2}(\hat{r}, \hat{t})}{\partial \hat{r}^{4}}+\frac{2}{\hat{r}} \frac{\partial^{3} \hat{w}_{2}(\hat{r}, \hat{t})}{\partial \hat{r}^{3}}-\frac{1}{\hat{r}^{2}} \frac{\partial^{2} \hat{w}_{2}(\hat{r}, \hat{t})}{\partial \hat{r}^{2}}\right.} \\
& \left.+\frac{1}{\hat{r}^{3}} \frac{\partial \hat{w}_{2}(\hat{r}, \hat{t})}{\partial \hat{r}}\right]+\hat{c} \frac{\partial \hat{w}_{1}(\hat{r}, \hat{t})}{\partial \hat{t}}+\left(\rho_{1} h_{1}\right) \frac{d^{2} \hat{w}_{1}}{d \hat{t}^{2}} \\
& =\frac{\left(V_{d}+V_{a} \cos (\hat{\Omega} \hat{t})\right)^{2}}{2\left(d+\hat{w}_{1}\right)^{2}},
\end{aligned}
$$

where $\hat{w}_{2}$ is the deflection of the microplate in the one-layered section of microplate. Thus, the equation governing the system is defined as follows:

$$
\begin{aligned}
& {\left[\frac{\partial^{4} \hat{w}_{1}(\hat{r}, \hat{t})}{\partial \hat{r}^{4}}+\frac{2}{\hat{r}} \frac{\partial^{3} \hat{w}_{1}(\hat{r}, \hat{t})}{\partial \hat{r}^{3}}-\frac{1}{\hat{r}^{2}} \frac{\partial^{2} \hat{w}_{1}(\hat{r}, \hat{t})}{\partial \hat{r}^{2}}\right.} \\
& \left.+\frac{1}{\hat{r}^{3}} \frac{\partial \hat{w}_{1}(\hat{r}, \hat{t})}{\partial \hat{r}}\right]\left(\frac{A_{11} F_{11}-B_{11}^{2}}{A_{11}}\right) \\
& +\hat{c} \frac{\partial \hat{w}_{1}(\hat{r}, \hat{t})}{\partial \hat{t}}+\left(\rho_{1} h_{1}+\rho_{2} h_{2}\right) \frac{d^{2} \hat{w}_{1}}{d \hat{t}^{2}} \\
& =\frac{\left(V_{d}+V_{a} \cos (\hat{\Omega} \hat{t})\right)^{2}}{2\left(d+\hat{w}_{1}\right)^{2}} \quad 0 \prec \hat{r} \prec R_{i}, \\
& \frac{E_{1} h_{1}^{3}}{2}\left[\frac{\partial^{4} \hat{w}_{2}(\hat{r}, \hat{t})}{\partial \hat{r}^{4}}+\frac{2}{\hat{r}} \frac{\partial^{3} \hat{w}_{2}(\hat{r}, \hat{t})}{\partial \hat{r}^{3}}-\frac{1}{\hat{r}^{2}} \frac{\partial^{2} \hat{w}_{2}(\hat{r}, \hat{t})}{\partial \hat{r}^{2}}\right. \\
& \left.+\frac{1}{\hat{r}^{3}} \frac{\partial \hat{w}_{2}(\hat{r}, \hat{t})}{\partial \hat{r}}\right]+\hat{c} \frac{\partial \hat{w}_{2}(\hat{r}, \hat{t})}{\partial \hat{t}}+\left(\rho_{1} h_{1}\right) \frac{d^{2} \hat{w}_{2}}{d \hat{t}^{2}} \\
& =\frac{\left(V_{d}+V_{a} \cos (\hat{\Omega} \hat{t})\right)^{2}}{2\left(d+\hat{w}_{2}\right)^{2}}, \quad R_{i} \prec \hat{r} \prec R_{o} .
\end{aligned}
$$

For analytical convenience, the following changes the in variable are applied to the equation of motion.

$$
w_{i}=\frac{-\hat{w}_{i}}{d}, \quad i=1,2, \quad r=\frac{\hat{r}}{R}, \quad t=\frac{\hat{t}}{T},
$$

where:

$$
T=R_{0}^{2}\left(\frac{\rho h_{1}}{D}\right)^{0.5}, \quad D=\frac{E h_{1}^{3}}{12\left(1-v^{2}\right)} .
$$

In other words, the displacement term in the governing equation is normalized with respect to the initial gap height between the plates and electrode plate, the radial position term $r$ normalized concerning the plate radius, and time is normalized with respect to the constant $T$. The efficiency of these variables indicates that the maximum value for displacement, i.e., contact between microplate and the electrode, and the maximum value for the coordinate system along the radius are equal to one. After changing these variables, the following set of non-dimensional parameters is obtained: 


$$
\begin{aligned}
& c=\frac{R_{0}^{4}}{12\left(1-v^{2}\right)\left(\rho_{1} h_{1}^{5} D\right)^{0.5}} \hat{c}, \quad \Omega=R_{0}^{2}\left(\frac{\rho h_{1}}{D}\right)^{2} \hat{\Omega} \\
& D^{\prime}=\frac{A_{11} F_{11}-B_{11}^{2}}{A_{11}}, \quad D=\frac{E h_{1}^{3}}{12\left(1-v^{2}\right)} .
\end{aligned}
$$

By substituting Eqs. (14)-(16) into Eq. (13), the dimensionless form of the motion equation is as follows:

$$
\begin{gathered}
{\left[\frac{\partial^{4} w_{1}(r, t)}{\partial r^{4}}+\frac{2}{r} \frac{\partial^{3} w_{1}(r, t)}{\partial r^{3}}-\frac{1}{r^{2}} \frac{\partial^{2} w_{1}(r, t)}{\partial r^{2}}\right.} \\
\left.+\frac{1}{r^{3}} \frac{\partial w_{1}(r, t)}{\partial r}\right]\left(\frac{A_{11} F_{11}-B_{11}^{2}}{A_{11}}\right) \\
+c \frac{\partial w_{1}(r, t)}{\partial t}+\left(\frac{D h_{2}}{D^{\prime} h_{1}}\right) \frac{d^{2} w_{1}}{d t^{2}} \\
=\frac{2 D^{2} d^{3}}{D^{\prime} R^{4}} \frac{\left(V_{d}+V_{a} \cos (\Omega t)\right)^{2}}{2\left(1+w_{1}\right)^{2}}, \\
\quad 0 \prec r \prec \frac{R_{i}}{R_{o}}, \\
{\left[\frac{\partial^{4} w_{2}(r, t)}{\partial r^{4}}+\frac{2}{r} \frac{\partial^{3} w_{2}(r, t)}{\partial r^{3}}-\frac{1}{r^{2}} \frac{\partial^{2} w_{2}(r, t)}{\partial r^{2}}\right.} \\
\left.+\frac{1}{r^{3}} \frac{\partial w_{2}(r, t)}{\partial r}\right]+c \frac{\partial w_{2}(r, t)}{\partial t}+\frac{d^{2} w_{2}}{d t^{2}} \\
=\frac{2 D d^{3}}{R^{4}} \frac{\left(V_{d}+V_{a} \cos (\Omega t)\right)^{2}}{2\left(1-w_{2}\right)^{2}}, \quad \frac{R_{i}}{R_{o}} \prec r \prec 1 .
\end{gathered}
$$

To simplify the equations, the term $\nabla^{4} w_{i}(r, t)$ can be used, which is defined as follows:

$$
\begin{aligned}
& \nabla^{4} w_{i}(r, t) \\
& \quad=\left[\frac{\partial^{4} w_{i}(r, t)}{\partial r^{4}}+\frac{2}{r} \frac{\partial^{3} w_{i}(r, t)}{\partial r^{3}}-\frac{1}{r^{2}} \frac{\partial^{2} w_{i}(r, t)}{\partial r^{2}}\right. \\
& \left.\quad+\frac{1}{r^{3}} \frac{\partial w_{i}(r, t)}{\partial r}\right] \quad i=1,2 .
\end{aligned}
$$

\section{Static deflection}

Static equation is solved by Galerkin method. To this end, comparison functions are required. These functions are considered the mode shapes of a linear system, given the non-uniformity of cross-section. To obtain the mode shape of the non-uniform microplate, deflection is considered as $w_{1}(r, t)=\varphi_{1}(r) e^{i \bar{w}_{i} t}$ and $w_{2}(r, t)=\varphi_{2}(r) e^{i \bar{w}_{i} t}$, where $\varphi_{1 i}(r)$ is the function of the mode shape in the two-layered section and $\varphi_{2 i}(r)$ is the function of the mode shape in the singlelayer section. Moreover, $\bar{w}_{i}$ is the natural frequency of free vibration and $i$ indicates the mode number.
By substituting these assumptions into Eq. (17) and setting the external electrostatic force and damping effect equal to zero, the differential equation governing the mode shape is as follows:

$$
\begin{aligned}
& -\bar{\omega}^{2}+\frac{D h_{2}}{D^{\prime} h_{1}} \nabla^{4} \varphi_{1 i}(r)=0, \quad 0 \prec r \prec \frac{R_{i}}{R_{o}}, \\
& -\bar{\omega}^{2}+\nabla^{4} \varphi_{2 i}(r)=0, \quad \frac{R_{i}}{R_{o}} \prec r \prec 1 .
\end{aligned}
$$

Eq. (19) is Bessel's equation and its solution is as follows:

$$
\begin{aligned}
\varphi_{2 i}(r)= & C_{1} J_{m}\left(r \bar{\omega}_{i} \sqrt{\frac{D h_{2}}{D^{\prime} h_{1}}}\right)+C_{2} Y_{m}\left(r \bar{\omega}_{i} \sqrt{\frac{D h_{2}}{D^{\prime} h_{1}}}\right) \\
& +C_{3} I_{m}\left(r \bar{\omega}_{i} \sqrt{\frac{D h_{2}}{D^{\prime} h_{1}}}\right)+C_{4} K_{m} \\
& \left(r \bar{\omega}_{i} \sqrt{\frac{D h_{2}}{D^{\prime} h_{1}}}\right)=0, \quad 0 \prec r \prec \frac{R_{i}}{R_{o}}, \quad(20) \\
\varphi_{2 i}(r)= & C_{5} J_{m}\left(r \bar{\omega}_{i}\right)+C_{6} Y_{m}\left(r \bar{\omega}_{i}\right)+C_{7} I_{m}\left(r \bar{\omega}_{i}\right) \\
& +C_{8} K_{m}\left(r \bar{\omega}_{i}\right)=0, \quad \frac{R_{i}}{R_{o}} \prec r \prec 1,
\end{aligned}
$$

where $J$ is Bessel's function of the first kind, $Y$ is Bessel's function of the second kind, $I$ is the refined Bessel function of the first kind, and $K$ is the refined Bessel function of the second kind.

To obtain the unknown coefficients, boundary and continuity conditions at $R_{i}$ are used, as shown in Figure 3 . These boundary conditions are as follows:

$$
\begin{gathered}
\left.w_{1}(\hat{r})\right|_{\hat{r}=0}=\text { finite, }\left.\quad w_{2}(\hat{r})\right|_{\hat{r}=R_{o}}=0, \\
\left.w_{2}^{\prime}(\hat{r})\right|_{\hat{r}=R_{o}}=0,
\end{gathered}
$$

and the continuity conditions are:

$$
\begin{aligned}
& M_{1}=M_{2}, \quad N_{1}=N_{2}, \quad Q_{1}=Q_{2}, \\
& w_{1}\left(R_{i}\right)=w_{2}\left(R_{i}\right), \quad w_{1}^{\prime}\left(R_{i}\right)=w_{2}^{\prime}\left(R_{i}\right),
\end{aligned}
$$

where, as shown in Figure $3, M_{1}\left(N_{1}\right)\left(Q_{1}\right)$ and $M_{2}\left(N_{2}\right)\left(Q_{2}\right)$ are the moments (normal force)(shear

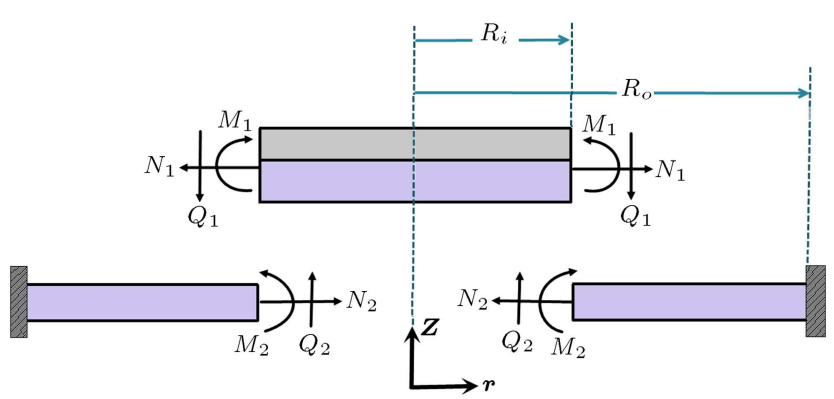

Figure 3. Free body diagram at the boundary between single- and two-layered sections. 
force) in the cross-section, which connect two-layered and single-layered parts; after applying Eqs. (21) and (22) into Eq. (20) and setting the determinant of coefficient equal to zero, the natural frequency and mode shapes are obtained. Now, by putting time derivatives equal to zero in Eq. (17), the equation governing static deflection is as follows:

$$
\begin{aligned}
& \nabla^{4} w_{s 1}(r)=\frac{2 D^{2} d^{3}}{D^{\prime} R^{4}} \frac{V_{d}^{2}}{\left(1-w_{s 1}(r)\right)^{2}}, \\
& \nabla^{4} w_{s 1}(r)\left(1-w_{s 1}(r)\right)^{2}=\frac{2 D^{2} d^{3}}{D^{\prime} R^{4}} V_{d}^{2}, \quad 0 \prec r \prec \frac{R_{i}}{R_{o}}, \\
& \nabla^{4} w_{s 2}(r)=\frac{2 D d^{3}}{R^{4}} \frac{V_{d}^{2}}{\left(1-w_{s 2}(r)\right)^{2}}, \\
& \nabla^{4} w_{s 2}(r)\left(1-w_{s 2}(r)\right)^{2}=\frac{2 D d^{3}}{R^{4}} V_{d}^{2}, \quad \frac{R_{i}}{R_{o}} \prec r \prec 1,(23)
\end{aligned}
$$

where $w_{s 1}$ indicates the static deflection in the twolayered section, and $w_{s 2}$ indicates the static deflection in the single-layered section of the microplate. The solution of Eq. (23) is assumed to be $w_{s 1}(r)=\sum_{i=1}^{m} a_{i} \varphi_{1 i}$ and $w_{s 2}(r)=\sum_{i=1}^{m} a_{i} \varphi_{2 i}$, where $\varphi_{1 i}$ and $\varphi_{2 i}$ are obtained in the former relation, $m$ is the number of modes for solving the equation, and $a_{i}$ is the unknown coefficient, obtained by applying the Galerkin method. Now, the considered assumption is substituted into Eq. (23) and, then, is multiplied by $\varphi_{1 i} j=1 . . m$. Next, the outcome is integrated with $r=0 \ldots 1$ that produces the following equations:

$$
\begin{aligned}
& \left(\int_{0}^{\frac{R_{i}}{R_{o}}}\left(1-\sum_{i=1}^{m} a_{i} \varphi_{1 i}\right)^{2}\left(a \nabla^{4} \varphi_{1 i}(r)\right) \varphi_{1 j}(r) d r\right) \\
& \quad-\frac{2 D^{2} d^{3}}{D^{\prime} R^{4}} V_{d}^{2} \int_{0}^{\frac{R_{i}}{R_{o}}} \varphi_{1 j}(r) d r+\int_{\frac{R_{i}}{R_{o}}}^{1}\left(1-\sum_{i=1}^{m} a_{i} \varphi_{2 i}\right)^{2} \\
& \left.\left(a_{i} \nabla^{4} \varphi_{2 i}(r)\right) \varphi_{2 j}(r) d r\right)-\frac{2 D d^{3}}{R^{4}} V_{d}^{2} \int_{\frac{R_{i}}{R_{o}}}^{1} \varphi_{2 j}(r) d r=0 .
\end{aligned}
$$

\section{Natural frequency of vibration about static deflection}

In Eq. (17), $w_{i}$ can be considered as the sum of dynamic deflection $\left(w_{d i}(r, t)\right)$ and static deflection $\left(w_{s i}(r)\right)$. Thus, by putting $\hat{w}_{1}(r, t)=w_{d 1}(r, t)+w_{s 1}(r)$ in the first relation and $\hat{w}_{2}(r, t)=w_{d 2}(r, t)+w_{s 2}(r)$ in the second relation of Eq. (17) and expanding electrostatic force around static position $w_{s i}(r)$, one obtains the following:

$$
\begin{aligned}
& \nabla^{4} w_{d 1}(r, t)+\frac{D h_{2}}{D^{\prime} h_{1}} \frac{\partial^{2} w_{d 1}(r, t)}{\partial t^{2}}+\frac{C D h_{2}}{D^{\prime} h_{1}} \frac{\partial w_{d 1}(r, t)}{\partial t} \\
& =\frac{2 D^{2} d^{3}}{D^{\prime} R^{4}}\left(\frac{2 V_{d} V_{a}+\left(V_{a} \cos (\Omega t)\right)^{2}}{\left(1-w_{s 1}(r)\right)^{2}}\right) \\
& +\frac{2\left(V_{d}+V_{a} \cos (\Omega t)\right)^{2}}{\left(1-w_{s 1}(r)\right)^{3}} w_{d 1}(r, t) \\
& +\frac{3\left(V_{d}+V_{a} \cos (\Omega t)\right)^{2}}{\left(1-w_{s 1}(r)\right)^{4}} w_{d 1}^{2}(r, t) \\
& +\frac{4\left(V_{d}+V_{a} \cos (\Omega t)\right)^{2}}{\left(1-w_{s 1}(r)\right)^{5}} w_{d 1}^{3}(r, t), \\
& 0 \prec r \prec \frac{R_{i}}{R_{o}}, \\
& \nabla^{4} w_{d 2}(r, t)+\frac{\partial^{2} w_{d 2}(r, t)}{\partial t^{2}}+C \frac{\partial w_{d 2}(r, t)}{\partial t} \\
& =\frac{2 D^{2} d^{3}}{D^{\prime} R^{4}}\left(\frac{2 V_{d} V_{a}+\left(V_{a} \cos (\Omega t)\right)^{2}}{\left(1-w_{s 2}(r)\right)^{2}}\right) \\
& +\frac{2\left(V_{d}+V_{a} \cos (\Omega t)\right)^{2}}{\left(1-w_{s 2}(r)\right)^{3}} w_{d 2}(r, t) \\
& +\frac{3\left(V_{d}+V_{a} \cos (\Omega t)\right)^{2}}{\left(1-w_{s 2}(r)\right)^{4}} w_{d 2}^{2}(r, t) \\
& +\frac{4\left(V_{d}+V_{a} \cos (\Omega t)\right)^{2}}{\left(1-w_{s 2}(r)\right)^{5}} w_{d 2}^{3}(r, t), \\
& \frac{R_{i}}{R_{o}} \prec r \prec 1 .
\end{aligned}
$$

By considering Eq. (25), the equation of linear free vibration of the system about static deflection is as follows:

$$
\begin{aligned}
& \nabla^{4} w_{d 1}(r, t)+\frac{D h_{2}}{D^{\prime} h_{1}} \frac{\partial^{2} w_{d 1}(r, t)}{\partial t^{2}} \\
& =\frac{4 D d^{3}}{R^{4}}\left(\frac{V_{d}^{2}}{\left(1-w_{s 1}(r)\right)^{2}}\right) w_{d 1}(r, t), \quad 0 \prec r \prec \frac{R_{i}}{R_{o}}, \\
& \nabla^{4} w_{d 2}(r, t)+\frac{\partial^{2} w_{d 2}(r, t)}{\partial t^{2}} \\
& =\frac{4 D^{2} d^{3}}{D^{\prime} R^{4}}\left(\frac{V_{d}^{2}}{\left(1-w_{s 2}(r)\right)^{2}}\right) w_{d 2}(r, t), \quad \frac{R_{i}}{R_{o}} \prec r \prec 1 .
\end{aligned}
$$

To obtain the natural frequency of linear vibration of the system about static deflection, $\omega_{n}$, it is assumed that $w_{d 1}(r, t)=\sum_{n=1}^{m} \varphi_{1 n}(r) q_{n}(t)$ and $w_{d 2}(r, t)=$ 
$\sum_{n=1}^{m} \varphi_{2 n}(r) q_{n}(t)$, where $q_{n}(t)$ is the time coordinate function corresponding to the free vibration of the system. By substituting these assumptions into Eq. (26), multiplying the outcome by $\varphi_{1 m}$ and $\varphi_{2 m}$, and integrating them into the whole microplate radius, the differential equation that governs the natural frequency is obtained as follows:

$$
\begin{aligned}
& \frac{D h_{2}}{D^{\prime} h_{1}}\left(\int_{0}^{\frac{R_{i}}{R_{o}}} \sum_{i=1}^{N} \varphi_{1 n}(r) \varphi_{1 m}(r) d r\right) \frac{d^{2} q_{n}(t)}{d t^{2}} \\
& +\left(\int_{0}^{\frac{R_{i}}{R_{o}}} \sum_{i=1}^{N} \nabla^{4} \varphi_{1 n}(r) \varphi_{1 m}(r) d r-\frac{4 D d^{3}}{R^{4}}\right. \\
& \left.\frac{V_{d}^{2}}{\left(1-w_{s 1}(r)\right)^{2}}\left(\int_{0}^{\frac{R_{i}}{R_{o}}} \sum_{i=1}^{N} \varphi_{1 n}(r) \varphi_{1 m}(r) d r\right)\right) \\
& q_{n}(t)+\left(\int_{\frac{R_{i}}{R_{o}}}^{1} \sum_{i=1}^{N} \varphi_{2 n}(r) \varphi_{2 m}(r) d r\right) \frac{d^{2} q_{n}(t)}{d t^{2}} \\
& +\left(\int_{\frac{R_{i}}{R_{o}}}^{1} \sum_{i=1}^{N} \nabla^{4} \varphi_{2 n}(r) \varphi_{2 m}(r) d r-\frac{4 D^{2} d^{3}}{D^{\prime} R^{4}}\right. \\
& \left.\frac{V_{d}^{2}}{\left(1-w_{s 2}(r)\right)^{2}}\left(\int_{\frac{R_{i}}{R_{o}}}^{1} \sum_{i=1}^{N} \varphi_{2 n}(r) \varphi_{2 m}(r) d r\right)\right) \\
& q_{n}(t)=0, \quad m=1, . .,, N .
\end{aligned}
$$

Now, the natural frequency may be obtained by applying a similar process, which is used for obtaining the natural frequency of a lumped mass system.

\section{Dynamic solution at primary resonance}

The dynamic responses are considered to be $w_{d 1}(r, t)=$ $p(t) \varphi_{11}(r)$ and $w_{d 2}(r, t)=p(t) \varphi_{21}(r)$, where $p(t)$ is the time coordinate function. After substituting the dynamic response into Eq. (25), multiplying it by the mode shape, and integrating the outcome into the whole microplate radius, the following equation results are obtained as follows:

$$
\begin{aligned}
K_{1} p(t) & +K_{2} \ddot{p}(t)+K_{3} \dot{p}(t)+K_{4} V_{a} \cos (\Omega t) \\
& =K_{5} p(t) K_{6} p(t)^{2}+K_{7} p(t)^{3} \\
& +\left(\frac{K_{8}}{V_{a} \cos (\Omega t)}+K_{9}\right) p(t)\left(V_{a} \cos (\Omega t)\right)^{2} \\
& +\left(\frac{K_{10}}{V_{a} \cos (\Omega t)}+K_{11}\right) p(t)^{2}\left(V_{a} \cos (\Omega t)\right)^{2}
\end{aligned}
$$

$$
\begin{aligned}
& +\left(\frac{K_{12}}{V_{a} \cos (\Omega t)}+K_{13}\right) p(t)^{3}\left(V_{a} \cos (\Omega t)\right)^{2} \\
& +K_{14} V_{a} \cos (\Omega t)^{2},
\end{aligned}
$$

where the coefficients of $K_{i}$ are defined as follows:

$$
\begin{aligned}
& K_{1}=\int_{0}^{\frac{R_{i}}{R_{o}}} \varphi_{11}(r) \nabla^{4} \varphi_{11}(r) d r \\
& +\int_{\frac{R_{i}}{R_{o}}}^{1} \varphi_{12}(r) \nabla^{4} \varphi_{12}(r) d r, \\
& K_{2}=\int_{0}^{\frac{R_{i}}{R_{o}}} \frac{D h_{2}}{D^{\prime} h_{1}} \varphi^{2}{ }_{11}(r) d r+\int_{\frac{R_{i}}{R_{o}}}^{1} \varphi_{12}{ }^{2}(r) d r \\
& K_{3}=\int_{0}^{\frac{R_{i}}{R_{o}}} \frac{C D h_{2}}{D^{\prime} h_{1}} \varphi^{2}{ }_{11}(r) d r+\int_{\frac{R_{i}}{R_{o}}}^{1} C \varphi_{12}{ }^{2}(r) d r, \\
& K_{4}=\int_{0}^{\frac{R_{i}}{R_{o}}} \frac{D^{2} d^{2}}{D^{\prime} R^{4}}\left(\frac{2 V_{d}}{\left(1-w_{s 1}(r)\right)^{2}} \varphi_{11}(r)\right) d r \\
& +\int_{\frac{R_{i}}{R_{o}}}^{1} \frac{2 V_{d}}{\left(1-w_{s 1}(r)\right)^{2}} \varphi_{12}(r) d r, \\
& K_{5}=\int_{0}^{\frac{R_{i}}{R_{o}}} \frac{D^{2} d^{2}}{D^{\prime} R^{4}}\left(\frac{2 V^{2}{ }_{d}}{\left(1-w_{s 1}(r)\right)^{3}} \varphi_{11}{ }^{2}(r)\right) d r \\
& +\int_{\frac{R_{i}}{R_{o}}}^{1} \frac{2 V_{d}^{2}}{\left(1-w_{s 1}(r)\right)^{3}} \varphi^{2}{ }_{12}(r) d r, \\
& K_{6}=\int_{0}^{\frac{R_{i}}{R_{o}}} \frac{D^{2} d^{2}}{D^{\prime} R^{4}}\left(\frac{3 V_{d}^{2}}{\left(1-w_{s 1}(r)\right)^{4}} \varphi_{11}^{3}(r)\right) d r \\
& +\int_{\frac{R_{i}}{R_{o}}}^{1} \frac{3 V^{2}{ }_{d}}{\left(1-w_{s 1}(r)\right)^{4}} \varphi^{3}{ }_{12}(r) d r, \\
& K_{7}=\int_{0}^{\frac{R_{i}}{R_{o}}} \frac{D^{2} d^{2}}{D^{\prime} R^{4}}\left(\frac{4 V_{d}^{2}}{\left(1-w_{s 1}(r)\right)^{5}} \varphi_{11}{ }^{4}(r)\right) d r \\
& +\int_{\frac{R_{i}}{R_{o}}}^{1} \frac{4 V^{2}{ }_{d}}{\left(1-w_{s 1}(r)\right)^{5}} \varphi^{4}{ }_{12}(r) d r, \\
& K_{8}=\int_{0}^{\frac{R_{i}}{R_{o}}} \frac{D^{2} d^{2}}{D^{\prime} R^{4}}\left(\frac{4 V^{2}{ }_{d}}{\left(1-w_{s 1}(r)\right)^{3}} \varphi_{11}^{2}(r)\right) d r \\
& +\int_{\frac{R_{i}}{R_{o}}}^{1} \frac{4 V^{2}{ }_{d}}{\left(1-w_{s 1}(r)\right)^{3}} \varphi^{2}{ }_{12}(r) d r,
\end{aligned}
$$




$$
\begin{aligned}
& K_{9}=\int_{0}^{\frac{R_{i}}{R_{o}}} \frac{D^{2} d^{2}}{D^{\prime} R^{4}}\left(\frac{2}{\left(1-w_{s 1}(r)\right)^{3}} \varphi_{11}{ }^{2}(r)\right) d r \\
& +\int_{\frac{R_{i}}{R_{o}}}^{1} \frac{2}{\left(1-w_{s 1}(r)\right)^{3}} \varphi^{2}{ }_{12}(r) d r, \\
& K_{10}=\int_{0}^{\frac{R_{i}}{R_{o}}} \frac{D^{2} d^{2}}{D^{\prime} R^{4}}\left(\frac{6 V_{d}}{\left(1-w_{s 1}(r)\right)^{4}} \varphi_{11}^{3}(r)\right) d r \\
& +\int_{\frac{R_{i}}{R_{o}}}^{1} \frac{6 V_{d}}{\left(1-w_{s 1}(r)\right)^{4}} \varphi^{3}{ }_{12}(r) d r, \\
& K_{11}=\int_{0}^{\frac{R_{i}}{R_{o}}} \frac{D^{2} d^{2}}{D^{\prime} R^{4}}\left(\frac{3}{\left(1-w_{s 1}(r)\right)^{4}} \varphi_{11}^{3}(r)\right) d r \\
& +\int_{\frac{R_{i}}{R_{o}}}^{1} \frac{3}{\left(1-w_{s 1}(r)\right)^{4}} \varphi^{3}{ }_{12}(r) d r, \\
& K_{12}=\int_{0}^{\frac{R_{i}}{R_{o}}} \frac{D^{2} d^{2}}{D^{\prime} R^{4}}\left(\frac{8 V_{d}}{\left(1-w_{s 1}(r)\right)^{5}} \varphi_{11}^{4}(r)\right) d r \\
& +\int_{\frac{R_{i}}{R_{o}}}^{1} \frac{8 V_{d}}{\left(1-w_{s 1}(r)\right)^{5}} \varphi^{4}{ }_{12}(r) d r, \\
& K_{13}=\int_{0}^{\frac{R_{i}}{R_{o}}} \frac{D^{2} d^{2}}{D^{\prime} R^{4}}\left(\frac{4}{\left(1-w_{s 1}(r)\right)^{5}} \varphi_{11}^{4}(r)\right) d r \\
& +\int_{\frac{R_{i}}{R_{o}}}^{1} \frac{4}{\left(1-w_{s 1}(r)\right)^{5}} \varphi^{4}{ }_{12}(r) d r, \\
& K_{14}=\int_{0}^{\frac{R_{i}}{R_{o}}} \frac{D^{2} d^{2}}{D^{\prime} R^{4}}\left(\frac{1}{\left(1-w_{s 1}(r)\right)^{2}} \varphi_{11}(r)\right) d r \\
& +\int_{\frac{R_{i}}{R_{o}}}^{1} \frac{1}{\left(1-w_{s 1}(r)\right)^{2}} \varphi_{12}(r) d r .
\end{aligned}
$$

Assuming that nonlinear terms are of weaker order than linear terms, Eq. (28) can be solved by means of multiple scales method of perturbation theory. Therefore, it is assumed that:

$$
\begin{aligned}
P(t)= & \varepsilon p_{1}\left(T_{0}, T_{1}, T_{2}\right)+\varepsilon^{2} p_{2}\left(T_{0}, T_{1}, T_{2}\right) \\
& +\varepsilon^{3} p_{3}\left(T_{0}, T_{1}, T_{2}\right),
\end{aligned}
$$

where $T_{0}=t, T_{1}=\varepsilon t$, and $T_{2}=\varepsilon^{2} t$ are time scales, and $\varepsilon$ is a dimensionless bookkeeping parameter representing the order of terms. By using chain derivatives, it can be written that:

$$
\begin{aligned}
& \frac{d}{d t}=D_{0}+\varepsilon D_{1}+\varepsilon^{2} D_{2}, \\
& \frac{d^{2}}{d t^{2}}=D_{0}{ }^{2}+2 \varepsilon D_{0} D_{1}+\varepsilon^{2}\left(2 D_{0} D_{2}+D_{1}^{2}\right),
\end{aligned}
$$

where $D_{n}$ is the operator of $\frac{d}{d T_{n}} ; n=0,1,2$.

To strike a balance between the terms of Eq. (28), the coefficient $K_{3}$ resulting from viscous damping is considered to be of order $\varepsilon^{2}$; moreover, the AC actuation is considered to be of order $\varepsilon^{3}$. By substituting Eq. (30) into Eq. (28) and separating terms with an equal order of $\varepsilon$, the following relations are obtained:

order $\left(\varepsilon^{1}:\right)$

$$
\frac{d^{2}}{d T_{0}{ }^{2}} p_{1}\left(T_{0}, T_{1}, T_{2}\right)+\frac{K_{1}-K_{5}}{K_{2}} p_{1}\left(T_{0}, T_{1}, T_{2}\right)=0
$$

order $\left(\varepsilon^{2}:\right)$

$$
\begin{aligned}
& \frac{d^{2}}{d T_{0}{ }^{2}} p_{2}\left(T_{0}, T_{1}, T_{2}\right)+\frac{K_{1}-K_{5}}{K_{2}} p_{2}\left(T_{0}, T_{1}, T_{2}\right) \\
& =-2 \frac{d^{2}}{d T_{0} T} p_{1}\left(T_{0}, T_{1}, T_{2}\right)-\frac{K_{6}}{K_{2}} p_{1}{ }^{2}\left(T_{0}, T_{1}, T_{2}\right),
\end{aligned}
$$

$\operatorname{order}\left(\varepsilon^{3}:\right)$

$$
\begin{aligned}
& \frac{d^{2}}{d T_{0}{ }^{2}} p_{3}\left(T_{0}, T_{1}, T_{2}\right)+\frac{K_{1}-K_{5}}{K_{2}} p_{3}\left(T_{0}, T_{1}, T_{2}\right)= \\
& -2 \frac{d^{2}}{d T_{0} T_{2}} p_{1}\left(T_{0}, T_{1}, T_{2}\right)-\frac{d^{2}}{d T_{1}{ }^{2}} p_{1}\left(T_{0}, T_{1}, T_{2}\right) \\
& -2 \frac{d^{2}}{d T_{0} T_{1}} p_{2}\left(T_{0}, T_{1}, T_{2}\right)-\frac{K_{3}}{K_{2}} \frac{d}{d T_{0}} p_{1}\left(T_{0}, T_{1}, T_{2}\right) \\
& -\frac{K_{4}}{K_{2}} \frac{d}{d T_{0}}\left(\frac{1}{2} V_{a}\left(e^{i \Omega T_{0}}+e^{-i \Omega T_{0}}\right)\right) \\
& -\frac{2 K_{6}}{K_{2}} p_{1}\left(T_{0}, T_{1}, T_{2}\right) p_{2}\left(T_{0}, T_{1}, T_{2}\right) \\
& -\frac{K_{7}}{K_{2}} p^{3}{ }_{1}\left(T_{0}, T_{1}, T_{2}\right) .
\end{aligned}
$$

Assuming that $\omega=\frac{K_{1}-K_{5}}{K_{2}}$, the solution of Eq. (32) is presented as follows:

$$
p_{1}\left(T_{0}, T_{1}, T_{2}\right)=A\left(T_{1}, T_{2}\right) e^{i \omega T_{0}}+\bar{A}\left(T_{1}, T_{2}\right) e^{-i \omega T_{0}},
$$

where $A\left(T_{1}, T_{2}\right)$ is a complex coefficient that is obtained by applying the solvability condition by substituting Eq. (35) into Eq. (33): 


$$
\begin{aligned}
\frac{d^{2}}{d T_{0}{ }^{2}} p_{2} & \left(T_{0}, T_{1}, T_{2}\right)+\omega^{2} p_{2}\left(T_{0}, T_{1}, T_{2}\right)= \\
& -2 i \omega e^{i \omega T_{0}} \frac{d}{d T_{1}} A\left(T_{1}, T_{2}\right) \\
& +\frac{K_{6}}{K_{2}} A\left(T_{1}, T_{2}\right) e^{2 i \omega T_{0}} \\
& +\frac{2 K_{6}}{K_{2}} A\left(T_{1}, T_{2}\right) \bar{A}\left(T_{1}, T_{2}\right)+C C .
\end{aligned}
$$

$C C$ indicates a combination of complex terms. In the case of solvability condition, the coefficient of a secular term in Eq. (36), i.e., the coefficient of $e^{i \omega T_{0}}$, must become equal to zero:

$$
\frac{d}{d T_{1}} A\left(T_{1}, T_{2}\right) e^{i \omega T_{0}}+\frac{d}{d T_{1}} \bar{A}\left(T_{1}, T_{2}\right) e^{-i \omega T_{0}}=0 .
$$

It is concluded from Eq. (37) that $A$ should only be a function of $T_{2}$. A particular solution to Eq. (36) is obtained as follows:

$$
\begin{aligned}
p_{2}\left(T_{0}, T_{1}, T_{2}\right)= & \frac{K_{6}}{K_{2}}\left(-\frac{1}{3 \omega^{2}} A^{2}\left(T_{2}\right) e^{2 i \omega T_{0}}\right. \\
& \left.-\frac{2 A\left(T_{2}\right) \bar{A}\left(T_{2}\right)}{\omega^{2}}\right)+C C .
\end{aligned}
$$

Now, $p_{1}$ and $p_{2}$ from Eqs. (35) and (37) are substituted into Eq. (34), and the actuation frequency, variation is considered as $\Omega=\omega+\varepsilon^{2} \sigma$ where $\sigma$, is a detuning parameter. In what follows, the terms causing secular terms in equations are retained; thus, one obtains:

$$
\begin{aligned}
\frac{d^{2}}{d T_{0}{ }^{2}} p_{3}\left(T_{0}, T_{1}, T_{2}\right)+\omega^{2} p_{3}\left(T_{0}, T_{1}, T_{2}\right) \\
=-2 i \omega e^{i \omega T_{0}} \frac{d}{d T_{2}} A\left(T_{2}\right)-i \omega \frac{K_{3}}{K_{2}} A\left(T_{2}\right) e^{i \omega T_{0}} \\
+\frac{K_{4}}{K_{2}}\left(\frac{1}{2} V_{a}\left(e^{i \sigma T_{0}} e^{i \omega T_{0}}\right)\right) \\
+\left(-\frac{10\left(\frac{K_{6}}{K_{2}}\right)^{2}}{3 \omega^{2}}-3 \frac{K_{7}}{K_{2}}\right)+N S T+C C
\end{aligned}
$$

NST represents all terms that are not secular. The term with the coefficient of $e^{i \omega T_{0}}$ in Eq. (39) is a secular term. By setting it equal to zero and substituting $A\left(T_{2}\right)$ as polar, $\frac{1}{2} a\left(T_{2}\right) e^{i \beta\left(T_{2}\right)}$, and separating real part from imaginary one, the result will be as follows:

$$
\begin{aligned}
\frac{d}{d T_{2}} a\left(T_{2}\right)= & \frac{\mu}{2} a\left(T_{2}\right)-\frac{F V_{a}}{\omega} \sin \left(\gamma\left(T_{2}\right)\right), \\
\frac{d}{d T_{2}} \gamma\left(T_{2}\right)= & -\frac{1}{8 \omega} S a\left(T_{2}\right)^{2}+\frac{F V_{a}}{\omega a\left(T_{2}\right)} \\
& \cos \left(\gamma\left(T_{2}\right)\right)+a\left(T_{2}\right)^{2} \sigma^{2}
\end{aligned}
$$

where:

$$
\begin{aligned}
& F=\frac{K_{4} V_{a}}{2 K_{2} \omega}, \quad \gamma\left(T_{2}\right)=\sigma T_{2}-\beta\left(T_{2}\right), \\
& S=\left(-\frac{10\left(\frac{K_{6}}{K_{2}}\right)^{2}}{3 \omega^{2}}-3 \frac{K_{7}}{K_{2}}\right), \quad \mu=\frac{K_{3}}{K_{2}} .
\end{aligned}
$$

By setting $\frac{d}{d T_{2}} a\left(T_{2}\right)$ and $\frac{d}{d T_{2}} \gamma\left(T_{2}\right)$ equal to zero in Eq. (40), the equation governing equilibrium solution amplitude of $\left(a_{0}\right)$ will be as follows:

$$
a_{0}{ }^{2}\left[\left(\frac{\mu}{2}\right)^{2}+\left(\sigma-\frac{s a_{0}^{2}}{\omega}\right)^{2}\right]=\left(\frac{F}{2 \omega} V_{a}\right)^{2} .
$$

Eq. (42) demonstrates that the maximum amplitude of $a_{0}$ occurs when the term inside the second parentheses is equal to zero; thus,

$$
a_{0}=\frac{2 F}{\mu \omega}, \quad \sigma=\frac{s a_{0}^{2}}{\omega} .
$$

By considering Eq. (43), the nonlinear resonance frequency is as follows:

$$
\Omega=\omega+\varepsilon^{2} \frac{4 s F^{2}}{\omega^{3} \mu^{2}} .
$$

\section{Results and discussion}

In order to show the accuracy of the calculations, the thickness of the second layer is set equal to zero and the static deflection is compared with the results obtained by Vogl and Nayfeh [3]. This comparison shown in Figure 4 indicates efficient conformity amongst the results.

In the following, the effects of the changes of the second-layer radius and thickness while assuming the thickness and radius to remain constant, respectively, and the simultaneous effect of changes in the radius and thickness while assuming volume to remain unchanged

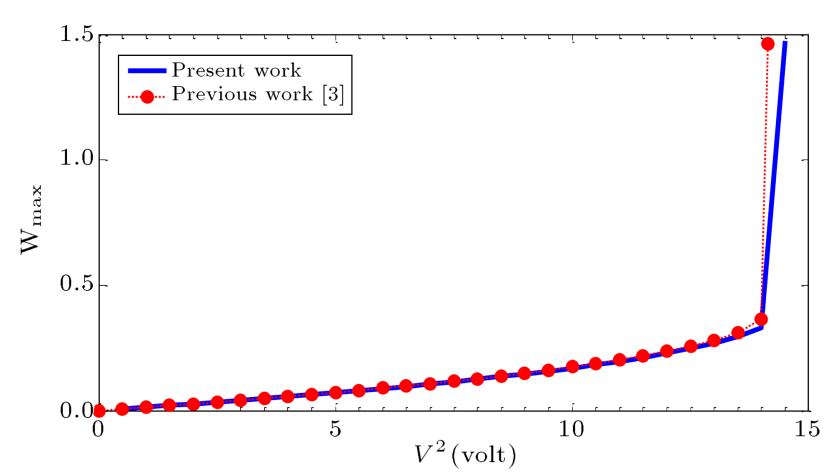

Figure 4. Static deflection variation of the center of the microplate against changes in electrostatic voltage for one-layered microplate, $h_{2}=0$. 


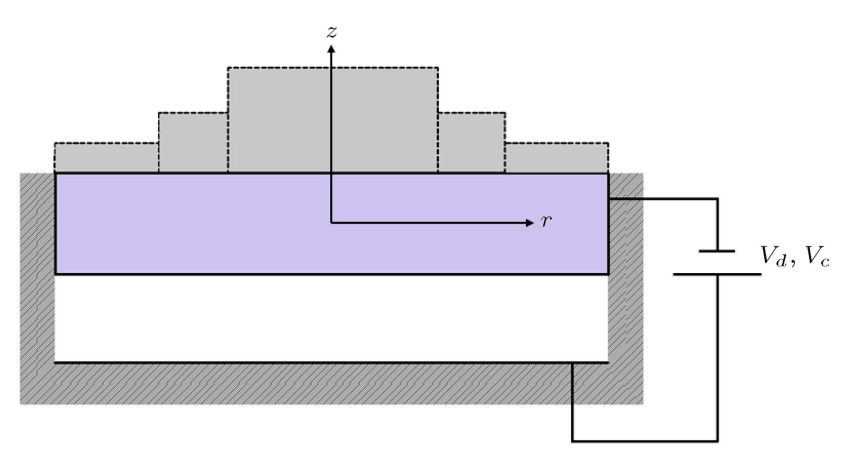

Figure 5. Increase of radius and decrease of thickness of the second layer when its volume remains constant.

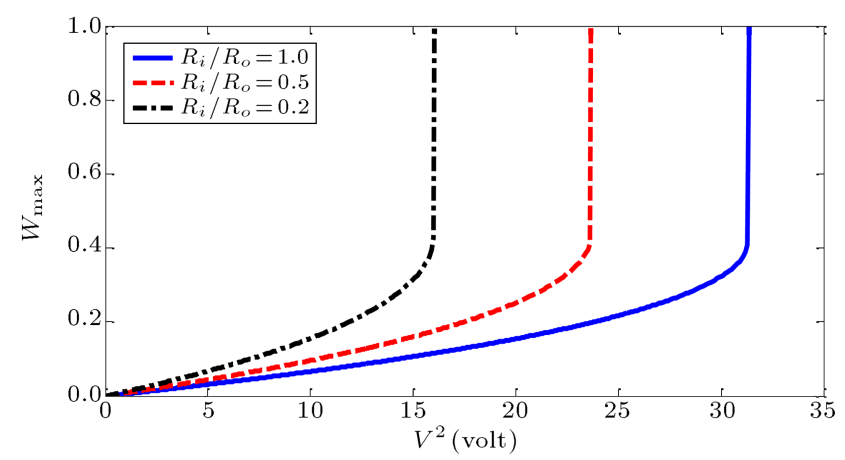

Figure 6. Variations of static deflection of the microplate center against the variation of electrostatic voltage for different values of the second-layer radius when the second-layer thickness remains constant $\left(h_{2} / h_{1}=2 / 14\right)$.

on the mechanical behavior of the system are investigated (see Figure 5). In this study, the parameters are considered according to Table 1.

Figure 6 shows variations of the static deflection at the microplate center for different values of the second-layer radius, assuming that its thickness remains constant. It is demonstrated that when the second layer is deposited on the entire area of the first layer, the system has the maximum static deflection and lowest pull-in voltage. The pull-in voltage is the voltage in which the slop of the curve tends to infinity. It is shown that with a decrease in the value of the second-layer radius, the static deflection increases while the pull-in voltage decreases. These changes result from the changes in flexural stiffness. It is obvious that an increase in the value in the second-layer radius causes an increase in the value of flexural stiffness, which in turn results in an increase in the value of static deflection. Moreover, since the electrostatic force on the right-hand side of Eq. (23) has $\left(1-w_{s}\right)^{2}$ in its denominator, i.e., with an increase in the value of static deflection, the value of electrostatic force

Table 1. Parameters of system.

\begin{tabular}{cccc}
\hline $\boldsymbol{E}$ & $\boldsymbol{h}_{\mathbf{1}}$ & $\boldsymbol{V}_{\boldsymbol{d}}$ & $\boldsymbol{V}_{\boldsymbol{a}}$ \\
\hline $169 \mathrm{GPa}$ & $140 \mu \mathrm{m}$ & $0.5 \mathrm{~V}$ & $0.03 \mathrm{~V}$ \\
\hline
\end{tabular}

increases as nonlinear. Therefore, variation changes of the static deflection and pull-in voltage show a nonlinear behavior.

Since the electrostatic force is nonlinear, the pull-in voltages have increased by $100 \%$ with a fivefold increase in the length of the second-layer radius, reaching $34 \mathrm{~V}$ from $17 \mathrm{~V}$. According to Figure 7 , the value of natural frequency goes up as the radius of the second-layer increases. Considering the linear terms of Eq. (28), these changes are in direct relationship with $K_{1}$ and $K_{5}$, resulting from the flexural stiffness and electrostatic force, respectively. For Eq. (29), the term $\left(1-w_{s i}\right)^{3}$ appears in the denominator of the equation governing on $K_{5}$. Since the static deflection decreases nonlinearly with an increase in the value of the second-layer radius, the natural frequency increases nonlinearly.

In Figure 8, the frequency response function is

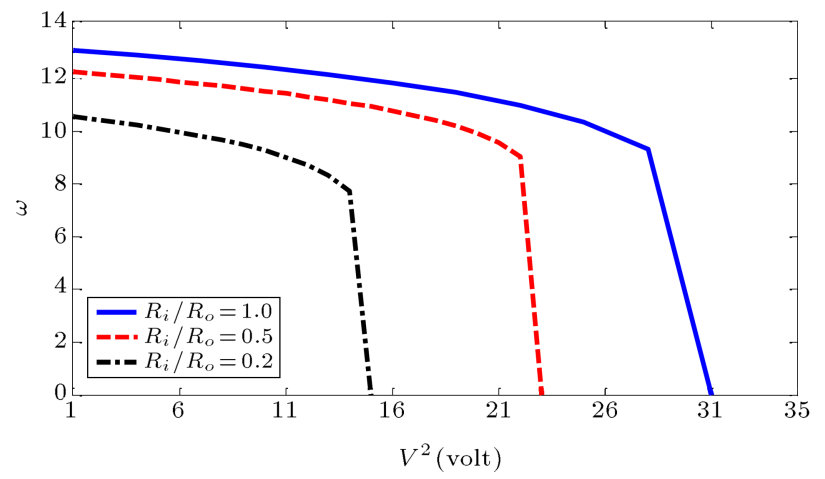

Figure 7. Variation of natural frequency of microplate against variation of electrostatic voltage for different values of the second-layer radius when second-layer thickness remains constant $\left(h_{2} / h_{1}=2 / 14\right)$.

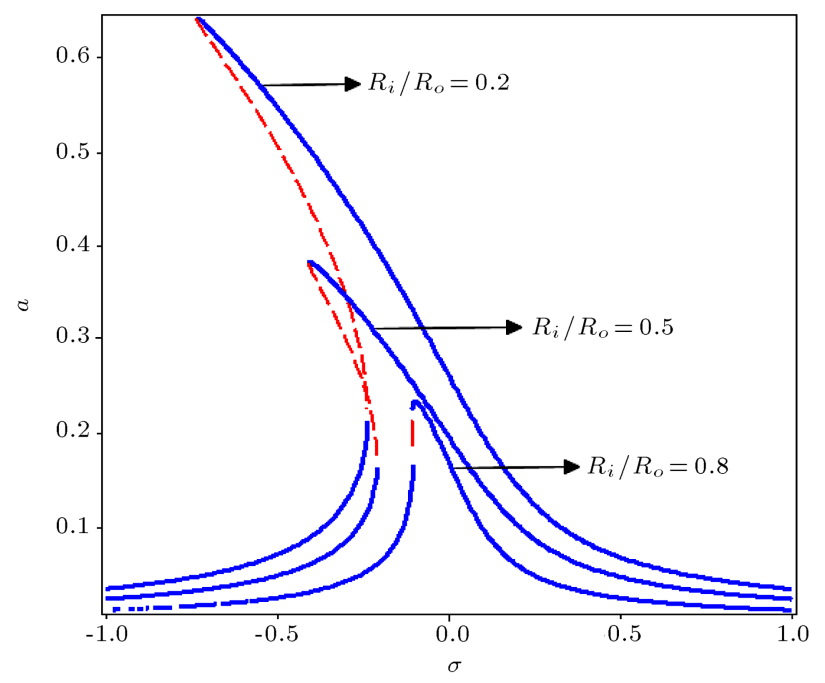

Figure 8. Variation of amplitude of steady state response against the variation of the detuning parameter for different values of the second-layer radius when thickness remains constant $\left(h_{2} / h_{1}=4 / 14, c=0.169\right)$. 


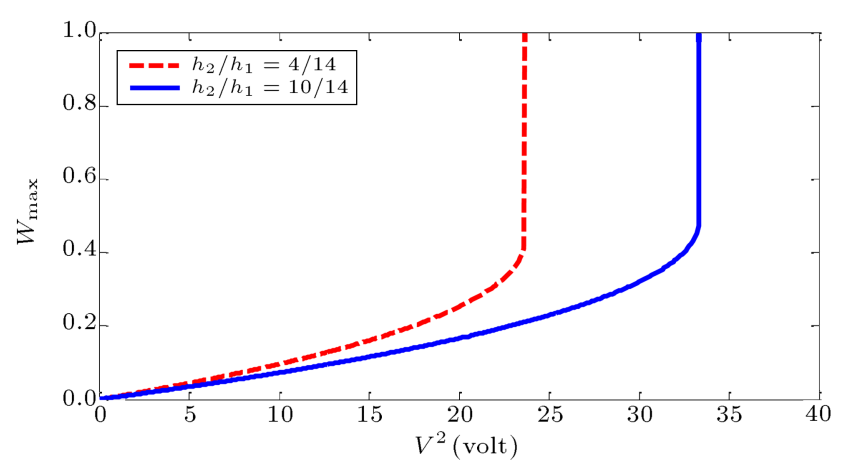

Figure 9. Changes in static deflection of the center of microplate against electrostatic voltage variations for different values of the second-layer thickness when its radius remains constant $\left(R_{i} / R_{0}=0.5\right)$.

shown for different values of the second-layer radius. It is shown that the value of amplitude peak and nonlinear shift of resonance decreases with an increase in the value of the second-layer radius. This reduction is caused by the decrease of the values of $S$ and $F$ and the increase of the value of natural frequency, which are the terms of Eqs. (42), (43), and (44). There are the terms $\left(1-w_{s}\right)^{2}, F\left(1-w_{s}\right)^{5}$, and $\left(1-w_{s}\right)^{4}$ in the denominator of the governing equation on $K_{4}, K_{7}$, and $K_{6}$. With an increase in the value of the second-layer radius, these terms are reduced due to the decrease of the value of static deflection. Therefore, considering Eq. (41), the parameter $S$ decreases, causing a decrease in the amplitude and nonlinear shift of resonance frequency.

Figure 9 shows the variations of the static deflection as the thickness of the second layer changes, assuming that its radius remains unchanged. It denotes that static deflection decreases with an increase in the value of the second-layer thickness, and pull-in voltage reaches $34 \mathrm{~V}$ from $24 \mathrm{~V}$ when the thickness increases 2.5 times. From Figure 10, the system's natural frequency increases with an increase in the value of the second-

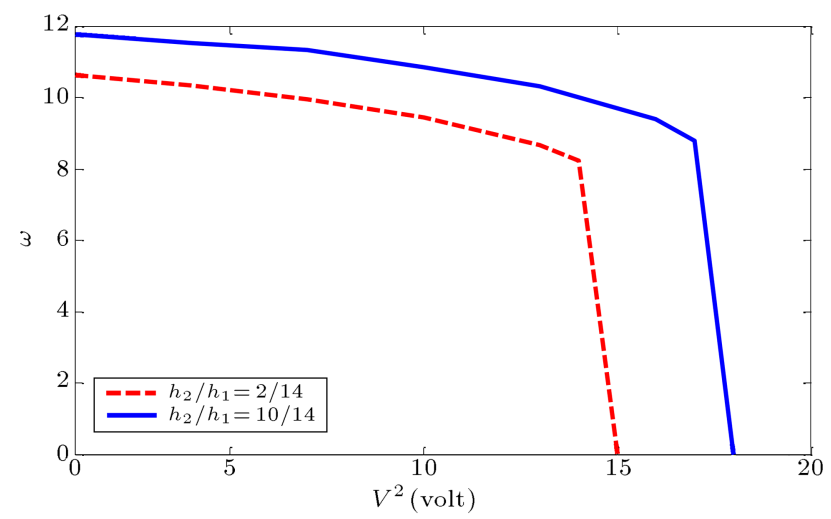

Figure 10. Variation of the natural frequency of the system against electrostatic voltage variations for different values of the second-layer thickness when its radius remains constant $\left(R_{i} / R_{0}=0.5\right)$. layer thickness, assuming that the radius of the second layer remains constant. Figure 11 shows the steady state vibration amplitude against changes between excitation frequency and natural frequency for different values of the second-layer radius. It is demonstrated that the non-linear shift of resonance frequency and the amplitude at resonance frequency decrease with an increase in the value of the second-layer thickness. These variations may be verified according to the discussion presented in previous paragraphs for verifying the mechanical behavior of the system due to variation of the second-layer radius.

To reach the most optimal possible mode, variations of static deflection and natural frequency are studied with the simultaneous change of radius and thickness of the second layer, assuming that its volume remains unchanged (see Figure 5). Firstly, it is assumed that the second layer is deposited on the whole area of the first layer; then, the radius and thickness of the second layer decrease and increase, respectively. Figures 12 and 13 demonstrate that static deflection, natural frequency, and the pull-in voltage decrease, increase, and increase, respectively. Then, by

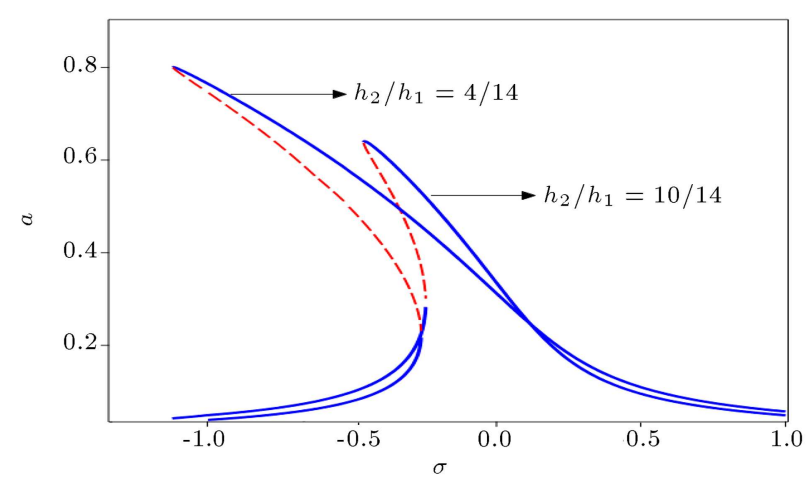

Figure 11. Variations of amplitude of steady state response against the variation of the detuning parameter for different values of the second-layer thickness when its radius remains constant $\left(R_{i} / R_{0}=0.5, c=0.169\right)$.

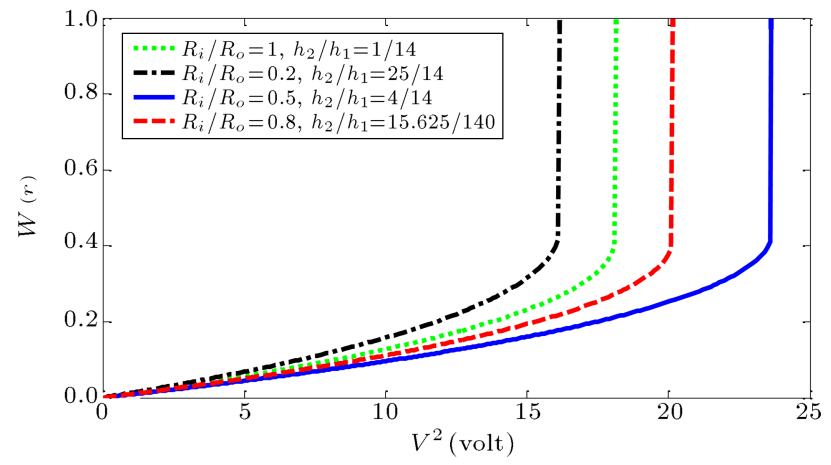

Figure 12. Variations of static deflection of the center of the microplate against changes in electrostatic voltage for the two-layered microplate with changes in radius and thickness when its volume remains constant. 


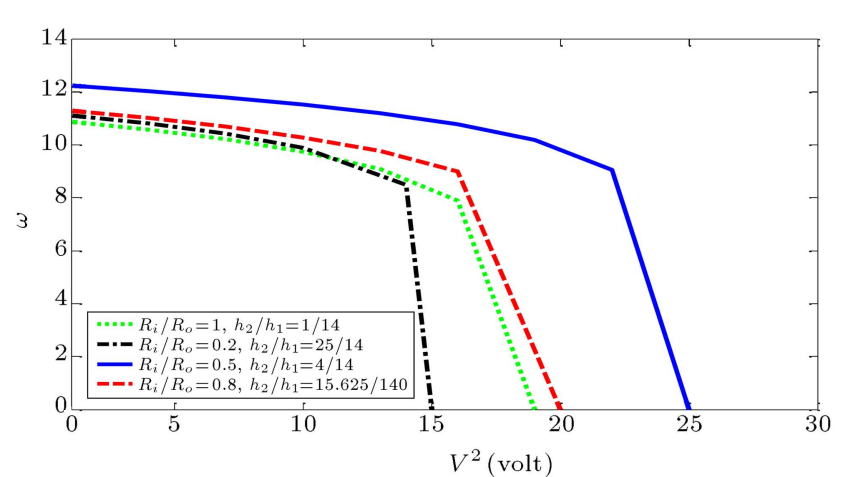

Figure 13. Variations of natural frequency against changes in electrostatic voltage for the two-layered microplate with changes in radius and thickness by assuming the volume remains constant.

exceeding a specific value of the second-layer radius, this behavior is reversed. These figures show that the maximum value of the natural frequency and the minimum value of static deflection occur when the second layer covers $50 \%$ of the first layer.

Figure 14 shows that when the second layer covers $20 \%$ of the first layer, by increasing the value of the second-layer radius to $50 \%$ of the first-layer radius, the amplitude and nonlinear shift of response frequency decrease; in addition, these parameters behave inversely once the second-layer radius value increases to $80 \%$. In fact, as the radius of the second layer decreases, flexural stiffness increases due to an increase in the thickness of the middle section of the microplate, which has an important role in equivalent bending stiffness in the system. According to Figures 6-8, increasing the bending stiffness decreases static deflection, increases natural frequency and pull-in voltage, and decreases the nonlinear natural shift of resonance frequency. As

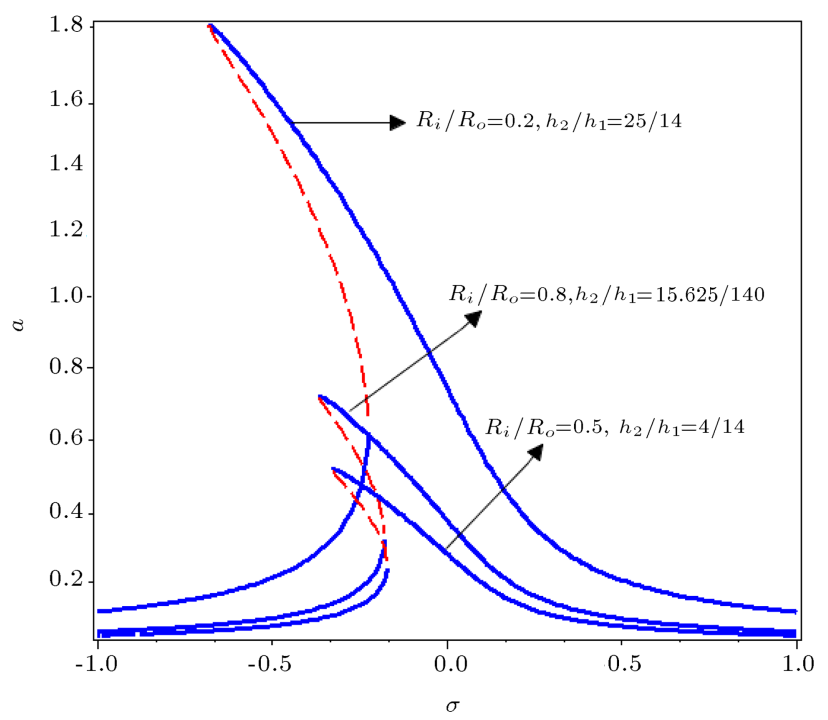

Figure 14. Variations of vibration amplitude considering the amount of changes in $\sigma$ with the variation of thickness and radius when the volume remains constant, $c=0.169$. the process goes on and the radius of the second layer decreases more, the second layer becomes like a concentrated mass in the middle of the microplate. In fact, the microplate acts like a one-layered circular plate with a concentrated mass in its center. Therefore, the variation of the mechanical behavior is reversed.

\section{Conclusion}

In the present study, the static deflection, natural frequency, pull-in voltage, and frequency response function of a two-layered clamped microplate subjected to electrostatic actuation were studied. First, non-linear equations of motion were derived based on the instructions of Classical Laminated Plate Theory (CLPT). Then, differential equations governing the static deflection and frequency of free vibration around the system static position were solved by means of Galerkin approach. Three mode shapes of a nonuniform clamped microplate were used as the comparison functions. Moreover, the equations of vibration around the static position were separated using Galerkin method and, then, solved by the multiple scale perturbation theory.

In this study, the mechanical behavior of the system was examined for different values of the radius and thickness of the second layer. It was observed that an increase in the value of the second-layer radius when its thickness remains constant and an increase in the value of the second-layer thickness when its radius remains constant led to an increase in the pull-in voltage and natural frequency while decreasing the static deflection, non-linear shift of resonance frequency, and steady static response amplitude.

It was shown that when the second layer was deposited on the whole area of the first layer, by decreasing the value and increasing the thickness of the first-layer radius when its volume remained constant, static deflection decreased and natural frequency increased before reaching a specific value of the secondlayer radius; then, this behavior of these parameters was reversed. The results showed that the highest frequency and lowest static deflection occurred when the second layer covered fifty percent of the first layer. This result can be used for designing high-speed microsensors.

\section{References}

1. Younis, M.I. MEMS, Linear and Nonlinear Statics and Dynamics, Springer, New York (2011).

2. Poloei, E., Zamanian, M., and Hosseini, S.A.A. "Nonlinear vibration analysis of an electrostatically excited micro cantilever beam coated by viscoelastic layer with the aim of finding the modified configuration", Structural Engineering and Mechanics, 61(2), pp. 193207 (2017). 
3. Vogl, G.W. and Nayfeh, A.H. "A reduced-order model for electrically actuated clamped circular plates", Journal of Micromechanics and Microengineering, 15(4), p. $684(2005)$

4. Vogl, G.W. and Nayfeh, A.H. "Primary resonance excitation of electrically actuated clamped circular plates", Nonlinear Dynamics, 47(1), pp. 181-192 (2007).

5. Liao, L.D., Chao, P.C., Huang, C.W., et al. "DC dynamic and static pull-in predictions and analysis for electrostatically actuated clamped circular microplates based on a continuous model", Journal of $\mathrm{Mi}$ cromechanics and Microengineering, 20(2), p. 025013 (2009).

6. Bertarelli, E., Colnago, A., Ardito, R., et al. "Modelling and characterization of circular microplate electrostatic actuators for micropump applications", In Thermal, Mechanical and Multi-Physics Simulation and Experiments in Microelectronics and Microsystems, 16th International Conference on, pp. 1-7, IEEE (2015).

7. Wang, K.F., Wang, B., and Zhang, C. "Surface energy and thermal stress effect on nonlinear vibration of electrostatically actuated circular micro-/nanoplates based on modified couple stress theory", Acta Mechanica, 228(1), pp. 129-140 (2017).

8. Shooshtari, A. and Dalir, M.A. "Nonlinear free vibration analysis of clamped circular fiber metal laminated plates", Scientia Iranica, Transactions B, Mechanical Engineering, 22(3), p. 813 (2015).

9. Khorshidi Paji, M., Dardel, M., Pashaei, M.H., et al. "The effect of radial force on pull-in instability and frequency of rigid core circular and annular plates subjected to electrostatic field", Scientia Iranica, 25(4), pp. 2111-2129 (2018).

10. Karimzadeh, A., Ahmadian, M.T., and Rahaeifard, M. "Effect of size dependency on in-plane vibration of circular micro-rings", Scientia Iranica, 24(4), pp. 1996-2008 (2017).

11. Dowlati, S., Rezazadeh, G., Afrang, S., et al. "An accurate study on capacitive microphone with circular diaphragm using a higher order elasticity theory", Latin American Journal of Solids and Structures, 13(4), pp. 590-609 (2016).

12. Shahriari, B., Zargar, O., Baghani, M, et al. "Free vibration analysis of rotating functionally graded annular disc of variable thickness using generalized differential quadrature method", Scientia Iranica, 25(2), pp. $728-740$ (2018).

13. Jallouli, A., Kacem, N., Bourbon, G., et al. "Pullin instability tuning in imperfect nonlinear circular microplates under electrostatic actuation", Physics Letters A, 380(46), pp. 3886-3890 (2016).
14. Saghir, S., Bellaredj, M.L., Ramini, A., et al. "Initially curved microplates under electrostatic actuation: theory and experiment", Journal of Micromechanics and Microengineering, 26(9), p. 095004 (2016).

15. Batra, R.C., Porfiri, M., and Spinello, D. "Vibrations and pull-in instabilities of microelectromechanical von Kármán elliptic plates incorporating the Casimir force", Journal of Sound and Vibration, 315(4), pp. 939-960 (2008).

16. Batra, R.C., Porfiri, M., and Spinello, D. "Reducedorder models for microelectromechanical rectangular and circular plates incorporating the Casimir force", International Journal of Solids and Structures, 45(11), pp. 3558-3583 (2008).

17. Wang, Y.G., Lin, W.H., Li, X.M, et al. "Bending and vibration of an electrostatically actuated circular microplate in presence of Casimir force", Applied Mathematical Modelling, 35(5), pp. 2348-2357 (2011).

18. Yang, W.D., Kang, W.B., and Wang, X. "Thermal and surface effects on the pull-in characteristics of circular nanoplate NEMS actuator based on nonlocal elasticity theory", Applied Mathematical Modelling, 43, pp. 321$336(2017)$.

19. Zhang, Y. "Large deflection of clamped circular plate and accuracy of its approximate analytical solutions", Science China Physics, Mechanics \& Astronomy, 59(2), p. 624602 (2016).

20. Medina, L., Gilat, R., and Krylov, S. "Bistable behavior of electrostatically actuated initially curved micro plate", Sensors and Actuators A: Physical, 248, pp. 193-198 (2016).

21. Medina, L., Gilat, R., and Krylov, S. "Modeling strategies of electrostatically actuated initially curved bistable micro plates", International Journal of Solids and Structures, 118, pp. 1-13 (2017).

22. Caruntu, D.I. and Oyervides, R. Frequency response reduced order model of primary resonance of electrostatically actuated MEMS circular plate resonators, Communications in Nonlinear Science and Numerical Simulation, 43, pp. 261-270 (2017).

23. Caruntu, D.I. and Oyervides, R. "Voltage response of primary resonance of electrostatically actuated MEMS clamped circular plate resonators", Journal of Computational and nonlinear dynamics, 11(4), p. 041021 (2016).

24. Vahdat, A.S., Rezazadeh, G., and Afrang, S. "Improving response of a MEMS capacitive microphone filtering shock noise", Microelectronics Journal, 42(5), pp. 614-621 (2011) .

25. Sharafkhani, N., Rezazadeh, G., and Shabani, R. "Study of mechanical behavior of circular FGM microplates under nonlinear electrostatic and mechanical shock loadings", Acta Mechanica, 223(3), pp. 579-591 (2012).

26. Shabani, R., Sharafkhani, N., Tariverdilo, S., et al. "Dynamic analysis of an electrostatically actuated circular micro-plate interacting with compressible fluid", Acta Mechanica, 224(9), pp. 20-25 (2013). 
27. Li, Z., Zhao, L., Ye, Z., Wang, H., et al. "Resonant frequency analysis on an electrostatically actuated microplate under uniform hydrostatic pressure", Journal of Physics D: Applied Physics, 46(19), p. 195108 (2013).

28. Nabian, A., Rezazadeh, G., Haddad-derafshi, M., et al. "Mechanical behavior of a circular micro plate subjected to uniform hydrostatic and non-uniform electrostatic pressure", Microsystem Technologies, 14(2), pp. $235-240$ (2008).

29. Tian-Jie, C. "Model to analyze micro circular plate subjected to electrostatic force", Sensors \& Transducers, 153(6), p. 129 (2013).

30. Mohammadi, V., Ansari, R., Shojaei, M, et al. "Size-dependent dynamic pull-in instability of hydrostatically and electrostatically actuated circular microplates", Nonlinear Dynamics, 73(3), pp. 1515-1526 (2013).

31. Ansari, R., Gholami, R., Shojaei, M.F, et al. "Surface stress effect on the pull-in instability of circular nanoplates", Acta Astronautica, 102, pp. 140-150 (2014).

32. Rashvand, K., Rezazadeh, G., Mobki, H., et al. "On the size-dependent behavior of a capacitive circular micro-plate considering the variable length-scale parameter", International Journal of Mechanical Sciences, 77, pp. 333-342 (2013).

33. Salehipour, H., Nahvi, H., and Shahidi, A.R. "Exact analytical solution for free vibration of functionally graded micro/nanoplates via three-dimensional nonlocal elasticity", Physica E: Low-dimensional Systems and Nanostructures, 66, pp. 350-358 (2015).

34. Liu, C.C. "The stability and non-linear vibration analysis of a circular clamped microplate under electrostatic actuation", Smart Science, 5(3), pp. 132-139 (2017).
35. Deshpande, M. and Saggere, L. "An analytical model and working equations for static deflections of a circular multi-layered diaphragm-type piezoelectric actuator", Sensors and Actuators A: Physical, 136(2), pp. 673-689 (2007).

\section{Biographies}

Reza Sepahvandi is an MSc Student that graduated from the Mechanical Engineering Department, Kharazmi University, Tehran, Iran. His research interests include nonlinear vibration and MEMS/NEMS.

Mehdi Zamanian was born in Nahavand, Iran in 1979. He received his BS degree from Razi University and $\mathrm{MS}$ and $\mathrm{PhD}$ degrees in Mechanical Engineering from Tarbiat Modares University, Tehran, Iran. He is currently an Associate Professor at the Mechanical Engineering Department of Kharazmi University, Tehran, Iran. His research interests include nonlinear vibration, MEMS devices, and vortex induced vibration.

Behnam Firouzi is an MSc student that graduated from the Mechanical Engineering Department, Kharazmi University, Tehran, Iran. His research interests are in the areas of nonlinear vibration, nonlinear dynamics, and MEMS devices.

Seyyed Ali Asghar Hosseini was born in Qom, Iran in 1978. He received his BS degree from Iran University of Science and Technology, and MS and PhD degrees from Tarbiat Modares University, Tehran, Iran. He is currently an Associate Professor at the Mechanical Engineering Department of Kharazmi University, Tehran, Iran. His research interests include nonlinear and random vibrations. 